\title{
Associação da atividade física de lazer com o consumo de bebidas alcoólicas em adultos
}

\section{Association of physical activity in leisure-time with alcohol consumption in adults}

\section{AUTORES \\ Dartel Ferrari de Lima1(iD) \\ Michael Pereira da Silva ${ }^{2}$ (D) \\ Oldemar Mazzardo Júnior ${ }^{1}$ (D) \\ Lohan Anguera $\mathrm{Lima}^{3}$ (D) \\ Adelar Aparecido Sampaio ${ }^{1}$ (D) \\ Olinda do Carmo Luiz ${ }^{4}$ D \\ 1 Universidade Estadual do Oeste do Paraná Departamento de Educação Física. Marechal Cândido Rondon, Paraná, Brasil. \\ 2 Universidade Federal do Paraná, Departamento de Educação Física. Curitiba, Paraná, Brasil. \\ 3 Santa Casa de Misericórdia de São Paulo. Departamento de Ortopedia e Traumatologia. São Paulo, São Paulo, Brasil; \\ 4 Universidade de São Paulo. Departamento de Medicina Preventiva. São Paulo, São Paulo, Brasil.}

\section{CONTATO}

Dartel Ferrari de Lima

dartelferrari07@gmail.com

UNIOESTE. Centro de Ciências Humanas,

Educação e Letras - CCHEL

Rua Pernambuco, no 1777, Centro, Marechal

Cândido Rondon, Paraná, Brasil.

CEP: 85960-000.

DOI

10.12820/rbafs.v.22n6p576-83

\section{(ल) $\Theta \Theta$}

Copyright: This is an open-access article distributed under the terms of the Creative Commons Attribution License ${ }^{\circledR}$, which permits unrestricted use, distribution, and reproduction in any medium, provided that the original author and source are credited.

\begin{abstract}
RESUMO
Evidências mostram que atividade física $(\mathrm{AF})$ pode se associar a níveis mais elevados de consumo de bebida alcóolica. Assim, esse estudo teve como objetivo verificar se existe ou não associação do nível de AF de lazer com o consumo de bebida alcóolica entre adultos de ambos os sexos. Dados secundários foram coletados do Ministério da Saúde (Vigitel) referente ao inquérito transversal realizado no ano de 2014, na cidade de Curitiba, PR, Brasil. A AF foi a variável de exposição e o consumo de bebida alcoólica a variável de desfecho. Participaram do estudo 1.504 adultos, sendo 557 homens com idade média de 51,7 $\pm 18,6$ anos e 947 mulheres com idade média de 55,7 $\pm 16,9$ anos. O nível de AF foi categorizado em inativo e ativo e o consumo de bebida alcóolica em regular e abusivo. Os resultados foram controlados por sexo, idade e escolaridade. Os adultos ativos tiveram risco pelo menos 1,5 vezes maior (Razão de Prevalência $(R P)=1,5$; IC95\%: 1,2-1,9) para o consumo regular de bebida alcoólica e pelo menos 1,3 vezes maior $(\mathrm{RP}=1,3$; IC95\%: 0,8-2,1) para o consumo abusivo, quando comparado aos inativos. Os homens ativos tiveram risco pelo menos 1,7 vezes maior $(\mathrm{RP}=1,7$; IC95\%: 1,2-2,5) para o consumo regular e 2,3 vezes maior $(\mathrm{RP}=2,3$; $\mathrm{IC} 95 \%: 0,9-5,7)$ para o consumo abusivo, enquanto mulheres ativas tiveram risco pelo menos 1,4 vezes maior $(\mathrm{RP}=$ 1,4; IC95\%: 1,1-1,8) para o consumo regular e 0,9 vezes maior ( $\mathrm{RP}=0,9$; IC95\%: 0,6-1,7) para o consumo abusivo. Os resultados apoiam a existência de uma associação positiva incongruente entre $o$ consumo de bebida alcoólica e a prática de $\mathrm{AF}$.
\end{abstract}

Keywords: Bebedeira; Transtornos induzidos por álcool; Alcoolismo; Exercício; Alcoólico.

\section{ABSTRACT}

However, recent studies indicated that higher levels of physical activity may be associated with higher levels of alcohol consumption. The objective of this study is to verify the association between physical activity level and abusive alcohol consumption of adults of both sexes. Secondary data were collected from the Ministry of Health/Vigitel/2014. The PA was the exposure variable and alcohol consumption as the outcome variable. Data were collected from the individual behavior analysis of 1,504 Brazilian adults, 557 men with a mean age of $51.7 \pm 18.6$ years and 947 women with a mean age of $55.7 \pm 16.9$ years from Curitiba city. Were categorized according the level of $P A$ (inactive and active) and alcohol consumption (regular use and abusive use). The results were controlled by gender, age and schooling. Actives had at least 1.5 times higher risk (Prevalence Ratio $(P R)=1.5 ; 95 \%$ CI: 1.2-1.9) for regular consumption of alcoholic beverages and at least 1.3 times higher risk ( $P R=1.3$; 95\%CI: 0.8-2.1) for abusive consumption, when compared to inactives. Active men had at least 1.7 times higher risk ( $P R=1.7 ; 95 \%$ CI: 1.2-2.5) for regular consumption and 2.3 times higher risk ( $P R=2.3$; 95\%CI: 0 9-5.7) for abusive consumption, while active women had at least 1.4 times higher risk ( $P R=1.4 ; 95 \% C I$ : 1.1-1.8) for regular consumption and 0.9 times higher risk $(P R=$ 0.9; 95\%CI: 0.6-1.7) for abusive consumption. For the examined sample, the results provide support for the existence of an incongruent positive association between regular alcohol consumption and PA practice.

Palavras-chave: Binge drinking; Alcohol-induced disorders; Alcoholism; Exercise; Alcoholics.

\section{Introdução}

A percepção de que as pessoas que adotam a prática de AF regular também tendem a adotar outros hábitos positivos para a saúde parece coerente. Algumas evidências mostram que indivíduos fisicamente ativos tendem a fumar menos e a consumir dietas mais saudáveis ${ }^{1-3}$.
Embora a AF seja considerada como um fator de proteção contra danos à saúde, cada vez mais evidências indicam que a prática de $\mathrm{AF}$ e o consumo de bebida alcoólica estão associados positivamente na população em geral ${ }^{1-5}$, embora os mecanismos envolvidos nessa associação e as consequências permanecem obscuros ${ }^{4,5}$. 
Dois estudos de base populacional encontraram relação positiva entre a $\mathrm{AF}$ e o consumo de bebida alcoólica. $\mathrm{O}$ estudo de revisão sistemática conduzido por Piazza-Gardner e Barry em $2012^{6}$ mostrou relação inversa para essa associação em todas as idades investigadas. O outro estudo, de Musselman e Rutledge ${ }^{7}$, avaliou 296 estudantes universitários de ambos os sexos. Categorizou o nível de AF dos participantes conforme a pontuação fornecida pela aplicação do Questionário Internacional de Atividade Física (IPAQ). Houve maior consumo de bebida alcoólica para aqueles com níveis de $\mathrm{AF}$ mais alto.

As evidências positivas de associação da $\mathrm{AF}$ com o consumo de bebida alcoólica ainda não demonstrou se a afinidade apresenta potencialidade para causar transtornos nocivos induzidos por uso de álcool ou para causar alguma forma de dependência. Essa é uma diferença importante, posto que os distúrbios nocivos do uso de álcool estão fortemente associados às taxas de morbidade e mortalidade da população em geral, vez que, o álcool é a droga mais utilizada no mundo e tem o seu consumo admitido e incentivado pela sociedade ${ }^{8}$.

Há ampla documentação científica demonstrando que o consumo excessivo e crônico de álcool mantém relação causal de morbidades ${ }^{9}$ capaz de alterar patologicamente os músculos, o fígado, o coração e o cérebro ${ }^{10}$. Atualmente mais de dois bilhões de pessoas no mundo consomem álcool regularmente e, no ano de 2012, cerca de 3,3 milhões de mortes foram atribuídas a esse consumo, o que equivale a $6 \%$ das mortes por todas as causas ${ }^{11}$. No Brasil, estima-se que $50 \%$ da população consome álcool regularmente e $12 \%$ consomem grande quantidade de uma só vez. A este consumo é atribuído $6,2 \%$ da carga total das doenças crônicas não transmissíveis ${ }^{12}$.

Existem vários mecanismos que podem estar subjacentes a esta associação positiva. Os traços de personalidade podem determinar maior propensão ao envolvimento simultaneamente com a AF e com o consumo de bebida alcoólica. Não obstante, modelos de circuitos neurais associados à recompensa podem ser ativados pelo consumo de bebida alcoólica, pela prática de AF ou pela ação simultânea de ambos ${ }^{12-13}$.

Experimentos com animais reproduzem esta suposição, onde animais expostos à $\mathrm{AF}$ foram mais atraídos ao consumo de bebida alcoólica do que seus pares sedentários ${ }^{14}$. Comportamentos ligados às regiões específicas do cérebro, como a impulsividade no córtex pré-frontal, podem influenciar para esta associação ${ }^{15}$, vez que, tanto a AF como a bebida alcoólica promovem a liberação de transmissores neuroquímicos gratificantes naquele local ${ }^{16}$. Para uma leitura mais detalhada sobre a relação entre AF e consumo de bebida alcóolica, recomenda-se a revisão sistemática de Piazza-Gardner e Barry ${ }^{6}$.

Além das implicações para a saúde, o efeito nocivo do álcool inflige perdas sociais e econômicas significativas sobre o indivíduo e a sociedade. À luz do crescimento global da população e do crescimento previsto para o consumo de bebida alcoólica no mundo, é esperado acréscimo do fardo das doenças associados a este consumo, a menos que ações preventivas sejam efetivadas. Destaca-se que em países em desenvolvimento, como o Brasil, as taxas de morbidade e mortalidade são maiores para cada litro de álcool consumido do que nos países desenvolvidos e até o momento, os esforços e os recursos disponíveis não foram suficientes para reduzir a carga imposta à saúde pública pelo uso nocivo de bebida alcoólica. Diante do exposto, o objetivo desse estudo foi verificar se existe ou não a associação entre nível de $\mathrm{AF}$ de lazer e o consumo de bebida alcoólica de adultos de ambos os sexos residentes na cidade de Curitiba.

\section{Métodos}

\section{Delineamento do estudo}

Para este estudo, foram examinados dados transversais de uma amostra de adultos, de ambos os sexos, com idade igual ou superior a 18 anos obtida pelo Sistema de Vigilância de Fatores de Risco e Proteção para Doenças Crônicas não Transmissíveis por Entrevista Telefônica (Vigitel) para o ano de 2014. O Vigitel apresenta métodos próprios que podem ser encontrados em publicação prévia ${ }^{17}$ e é administrado pelo $\mathrm{Mi}^{-}$ nistério da Saúde brasileiro desde o ano de 2006.

O Vigitel foi projetado para coletar dados específicos sobre os determinantes das doenças crônicas na população adulta nas 26 capitais dos estados e do Distrito Federal mediante entrevista por telefone fixo ${ }^{18}$. Na edição de 2014, o Vigitel sorteou 101.200 linhas e 62.786 foram elegíveis, totalizando 40.853 entrevistas completadas, sendo 15.521 envolvendo homens e 25.332 envolvendo mulheres. Para este estudo, foram selecionadas 1.504 entrevistas completas, envolvendo 557 homens e 947 mulheres residentes na cidade de Curitiba-PR. Pesos pós-estratificação foram utilizados para homogeneizar a amostra utilizando o método Rake.

O método Rake utiliza procedimentos interativos que levam em conta as sucessivas comparações entre as estimativas da distribuição de cada variável sociodemográfica na amostra Vigitel e na população total de 
cada cidade. Essas comparações culminam no encontro de pesos que, aplicados à amostra Vigitel, igualam sua distribuição sociodemográfica à distribuição estimada para a população total de cada cidade ${ }^{17}$.

A amostra foi estratificada por sexo, idade, escolaridade e população de referência (distribuição da variável nos Censos Demográficos de 2000 e 2010 e sua variação anual média no período intercensitário), como tentativa de equiparar a representação da população com e sem serviços de telefonia fixa.

\section{Variáveis investigadas}

A variável de exposição foi determinada pelo nível de AF individual (inativo e ativo) e a variável de desfecho pelo consumo de bebida alcoólica (regular e abusivo). As variáveis de controle foram sexo, idade (18-39 anos; 40-59 anos e 60 anos ou mais) e escolaridade (0 a 8 anos; 9 a 11 anos e 12 ou mais anos).

\section{Consumo de bebidas alcoólicas}

Foi considerado um consumidor moderado de álcool, o homem ou a mulher que relatou ao entrevistador o consumo de alguma quantidade de álcool durante a semana, conforme a questão: "O(a) sr(a) costuma consumir bebida alcoólica?”. Foi considerado consumidor abusivo de álcool, o homem que relatou o consumo de cinco ou mais doses de bebida alcoólica e a mulher que relatou o consumo de quatro ou mais doses em uma única ocasião, pelo menos uma vez nos últimos 30 dias, conforme resposta à questão: "Nos últimos 30 dias, o sr. chegou a consumir cinco ou mais doses de bebida alcoólica em uma única ocasião?" ou "Nos últimos 30 dias, a sra. chegou a consumir quatro ou mais doses de bebida alcoólica em uma única ocasião?”. Uma dose de bebida alcoólica corresponde a uma lata de cerveja, uma taça de vinho ou uma dose de qualquer bebida alcoólica destilada. A frequência semanal do consumo moderado e abusivo de bebida alcoólica foi agrupada em quatro categorias: 1 a 2 dias; 3 a 4 dias; 5 a 6 dias; todos os dias da semana. Abstêmios foram os participantes que relataram nenhum consumo de bebida alcoólica nos últimos 30 dias antecedentes à entrevista.

\section{Classificação do nível da atividade física de lazer}

O nível de AF foi classificado em duas categorias, ativo e inativo. Foi considerado ativo o participante que respondeu afirmativamente à questão: "Nos últimos três meses, o(a) sr.(a) praticou algum tipo de exercício físico ou esporte?”. Foi considerado inativo o participante que respondeu negativamente à questão apresentada anteriormente. $\mathrm{O}$ entrevistado pode escolher uma entre 16 opções de exercício físico ou esporte: caminhada, caminhada na esteira rolante, musculação, hidroginástica, ginástica em geral, natação, artes marciais e lutas, ciclismo e voleibol/futevôlei, danças; corrida, corrida na esteira rolante, ginástica aeróbia, futebol/futsal, basquetebol e tênis.

\section{Análise estatística}

A análise descritiva dos dados foi realizada mediante a distribuição de frequência relativa das variáveis, utilizando o comando de pesquisa "svy" (com fatores de ponderação) que permitiu atribuir peso pós-estratificados distinto a cada indivíduo. A medida de prevalência estimou a proporção de indivíduos adultos que consumiram bebida alcoólica em 2014. Para modelar a contagem dos dados foi realizada a regressão de Poisson para estimar as Razões de Prevalência e seus respectivos intervalos de confiança de $95 \%$, sendo ajustado por sexo, idade e escolaridade, com nível de significância de 5\%. Diferenças substanciais encontradas entre os sexos no consumo de bebida alcoólica e nos níveis de AF conduziram as análises separadas para cada sexo ${ }^{1-3,6}$. Todas as análises foram realizadas no pacote estatístico StataMP ${ }^{\circledR}$ 13.0.

\section{Aspectos éticos}

O projeto Vigitel foi aprovado pela Comissão Nacional de Ética em Pesquisa para Seres Humanos do Ministério da Saúde (Parecer 355.590, de 26/6/2013 - CAAE: 16202813.2.0000.0008). O consentimento livre e esclarecido foi obtido oralmente no momento do contato telefônico com os entrevistados. O banco de dados é público e está disponível em: <http://svs.aids.gov.br/ bases_vigitel_viva/vigitel.php> e não possibilita a identificação dos entrevistados, razão porque foi dispensado de apreciação por Comitê de Ética em Pesquisa, em conformidade com a Resolução do Conselho Nacional de Saúde (CNS) no 510, de 7 de abril de 2016.

\section{Resultados}

Foram analisados dados de 1.504 adultos com idade igual ou superior a 18 anos, sendo 557 homens com idade média de $51,7 \pm 18,6$ anos e 947 mulheres com idade média de 55,7 $\pm 16,9$ anos. A amostra utilizada neste estudo (1.504 adultos) apresentou poder de 0,83 para a identificação de razões de prevalência significativas $(p<0,05)$ iguais ou superiores a 1,35.

A Tabela 1 apresenta a distribuição da frequência ponderada de indivíduos conforme o consumo regular de bebida alcoólica, o nível de AF, a idade e o nível de escola- 
ridade. O percentual de consumidores de bebida alcoólica entre os homens foi 39,1\% (IC95\%: 37,9-40,3) para os inativos, 49,9\% (IC95\%: 45,1-48,1) para os ativos. 12,8\% (IC95\%: 11,5-13,9) entre as mulheres inativas e 22,0\% (IC95\%: 20,2-24,8) para as mulheres ativas. A frequência do consumo de bebida alcoólica foi maior para homens em todas as análises. Mais da metade dos consumidores beberam em intervalos de 1 a 2 dias por semana, sem linearidade definida de aumento da frequência do consumo com o aumento da idade e do nível de escolaridade.

A Tabela 2 apresenta a distribuição da frequência semanal do consumo abusivo de bebida alcoólica para os diferentes níveis de AF para ambos os sexos. Os homens apresentaram maiores percentuais de consumo de bebida alcoólica em frequência e quantidade quando comparados às mulheres. Aproximadamente, 33\% dos homens e $13 \%$ das mulheres relataram o consumo abusivo de bebida alcoólica no período investigado. Entre os participantes ativos que relatara o uso abusivo de bebida alcoólica, 38,7\% (IC95\%: 25,4-41,1) eram homens e 12,3\% (IC95\%: 9,1-15,6) eram mulheres.

A Tabela 3 mostra a associação do consumo regular e abusivo de bebida alcoólica com a participação de AF de lazer. Para o consumo regular de bebida alcoólica

Tabela 1 - Frequência relativa de adultos ( $\geq 18$ anos) que, nos últimos 30 dias consumiram bebida alcoólica, distribuídos conforme o nível de atividade física (lazer) e sexo, segundo a idade, nível de escolaridade e frequência semanal do consumo, Vigitel - Curitiba, 2014.

\begin{tabular}{|c|c|c|c|c|}
\hline \multirow[b]{2}{*}{ Variáveis } & \multicolumn{2}{|c|}{ Inativos } & \multicolumn{2}{|c|}{ Ativos } \\
\hline & $\begin{array}{l}\text { Homens } \\
\text { (IC95\%) }\end{array}$ & $\begin{array}{l}\text { Mulheres } \\
\text { (IC95\%) }\end{array}$ & $\begin{array}{l}\text { Homens } \\
\text { (IC95\%) }\end{array}$ & $\begin{array}{l}\text { Mulheres } \\
\text { (IC95\%) }\end{array}$ \\
\hline \multicolumn{5}{|l|}{ Consumo de álcool } \\
\hline Sim & $39,1(37,9-40,3)$ & $12,8(11,5-13,9)$ & $49,9(45,1-48,1)$ & $22,0(20,2-24,8)$ \\
\hline \multicolumn{5}{|c|}{ Frequência do consumo } \\
\hline 1 a 2 dias/semana & $70,8(63,1-79,4)$ & $71,8(66,9-74,8)$ & $60,2(54,2-63,8)$ & $71,8(68,1-74,8)$ \\
\hline 3 a 4 dias/semana & $10,4(9,1-11,5)$ & $13,1(7,2-9,3)$ & $19,3(16,8-21,5)$ & $15,2(14,2-16,1)$ \\
\hline 5 a 6 dias/semana & $7,2(5,1-11,9)$ & $5,4(4,6-6,2)$ & $7,3(5,7-10,6)$ & $4,3(3,3-5,3)$ \\
\hline Todos os dias & $11,6(10,2-12,8)$ & $9,7(6,1-13,5)$ & $13,2(10,1-14,5)$ & $8,7(7,5-9,9)$ \\
\hline \multicolumn{5}{|l|}{ Idade (anos) } \\
\hline 18 a 24 & $8,1(7,0-9,3)$ & - & $9,1(8,1-12,5)$ & $2,2(1,4-3,1)$ \\
\hline 25 a 34 & $12,8(11,7-13,9)$ & $5,4(4,1-6,5)$ & $15,0(13,2-17,2)$ & $4,3(3,1-5,2)$ \\
\hline 35 a 44 & $12,8(11,2-13,9)$ & $13,5(12,2-14,8)$ & $16,5(14,6-18,8)$ & $8,6(7,5-9,8)$ \\
\hline 45 a 54 & $17,5(16,4-18,6)$ & $10,8(9,6-11,9)$ & $16,1(15,2-20,1)$ & $23,9(22,3-25,0)$ \\
\hline 55 a 64 & $20,9(19,3-22,1)$ & $18,9(17,5-20,1)$ & $18,4(16,5-18,9)$ & $23,9(22,1-25,1)$ \\
\hline 65 e mais & $27,9(16,6-28,9)$ & $51,3(49,1-52,3)$ & $24,9(21,3-28,6)$ & $36,9(34,4-38,1)$ \\
\hline \multicolumn{5}{|l|}{ Anos de escolaridade } \\
\hline 0 a 8 & $31,4(30,1-32,6)$ & $48,7(47,1-49,8)$ & $15,8(7,9-9,8)$ & $21,7(20,8-22,8)$ \\
\hline 9 a 11 & $26,7(25,4-27,9)$ & $16,2(15,1-17,5)$ & $31,2(30,5-33,7)$ & $28,3(26,8-29,5)$ \\
\hline 12 e mais & $41,9(36,7-49,1)$ & $35,1(34,0-36,3)$ & $53,0(57,1-60,0)$ & $50,0(18,9-51,6)$ \\
\hline
\end{tabular}

IC $95 \%=$ Intervalo de confiança de 95\%.

Tabela 2 - Percentual de adultos ( $\geq 18$ anos) que, nos últimos 30 dias, consumiram quatro ou mais doses (mulheres) ou cinco ou mais doses (homens) de bebida alcoólica em uma mesma ocasião, conforme o nível de atividade física (lazer), sexo e frequência semanal do consumo com seus respectivos intervalos de confiança de 95\% (IC95\%), Vigitel - Curitiba, 2014.

\begin{tabular}{lcccc}
\hline \multicolumn{1}{c}{ Variáveis } & \multicolumn{2}{c}{ Inativos } & Ativos \\
\cline { 2 - 5 } & $\begin{array}{c}\text { Homens } \\
\text { (IC95\%) }\end{array}$ & $\begin{array}{c}\text { Mulheres } \\
\text { (IC95\%) }\end{array}$ & $\begin{array}{c}\text { Homens } \\
\text { (IC95\%) }\end{array}$ & $\begin{array}{c}\text { Mulheres } \\
\text { (IC95\%) }\end{array}$ \\
\hline $\begin{array}{l}\text { Consumo abusivo de álcool }{ }^{*} \\
\text { Sim }\end{array}$ & $32,6(31,2-33,8)$ & $16,2(14,8-18,1)$ & $38,7(25,4-41,1)$ & $12,3(9,1-15,6)$ \\
Frequência do consumo & $33,1(30,8-38,0)$ & $46,6(40,1-57,9)$ & $51,6(46,1-58,9)$ & $63,3(58,1-69,4)$ \\
1 a 2 dias/semana & $16,8(13,1-25,2)$ & $20,0(16,1-24,0)$ & $12,2(9,9-16,8)$ & $11,0(8,9-14,8)$ \\
3 a 4 dias/semana & $25,6(22,9-31,8)$ & $6,0(4,8-7,9)$ & $14,7(13,1-15,9)$ & $9,3(7,9-15,7)$ \\
5 a 6 dias/semana & $17,4(12,1-21,2)$ & $10,7(9,4-11,5)$ & $11,4(9,2-14,7)$ & $6,7(4,2-11,8)$ \\
Todos os dias & $7,1(6,0-8,3)$ & $16,7(15,1-18,3)$ & $10,1(8,8-13,9)$ & $9,7(6,9-12,0)$ \\
Não informou & & &
\end{tabular}

* Consumo abusivo= consumo de quatro doses ou mais para mulheres e cinco doses ou mais para homens em mesma ocasião. 
Tabela 3 - Razão de prevalência (RP) do consumo e do abuso de álcool segundo o nível de atividade física de adultos com idade igual ou superior a 18 anos, Vigitel - Curitiba, 2014.

\begin{tabular}{|c|c|c|c|c|c|c|c|c|c|c|c|c|c|}
\hline \multirow{2}{*}{\multicolumn{2}{|c|}{$\begin{array}{l}\text { Consumo regular } \\
\text { de álcool }\end{array}$}} & \multicolumn{4}{|c|}{ Geral } & \multicolumn{4}{|c|}{ Homens } & \multicolumn{3}{|c|}{ Mulheres } & \multirow[b]{2}{*}{$\mathrm{p}$} \\
\hline & & $\begin{array}{l}\text { RP Bruta } \\
\text { (IC 95\%) }\end{array}$ & $\mathrm{p}$ & $\begin{array}{l}\text { RP Ajustada a } \\
\quad \text { (IC 95\%) }\end{array}$ & $\mathrm{p}$ & $\begin{array}{l}\text { RP Bruta } \\
\text { (IC 95\%) }\end{array}$ & $\mathrm{p}$ & $\begin{array}{l}\text { RP Ajustada }{ }^{b} \\
\quad \text { (IC 95\%) }\end{array}$ & $\mathrm{p}$ & $\begin{array}{l}\text { RP Bruta } \\
\text { (IC 95\%) }\end{array}$ & $\mathrm{p}$ & $\begin{array}{l}\text { RP Ajustada }{ }^{b} \\
\text { (IC 95\%) }\end{array}$ & \\
\hline \multirow[b]{2}{*}{ NAF } & Inativo & 1 & & 1 & & 1 & & 1 & & 1 & & 1 & \\
\hline & Ativo & $\begin{array}{c}1,5 \\
1,2-1,9\end{array}$ & 0,001 & $\begin{array}{c}1,4 \\
1,1-1,8\end{array}$ & 0,001 & $\begin{array}{c}1,7 \\
1,2-2,5\end{array}$ & 0,005 & $\begin{array}{c}1,7 \\
1,2-2,4\end{array}$ & 0,051 & $\begin{array}{c}1,4 \\
1,1-1,8\end{array}$ & 0,033 & $\begin{array}{c}1,3 \\
1-1,7\end{array}$ & 0,005 \\
\hline \multirow{2}{*}{\multicolumn{2}{|c|}{$\begin{array}{l}\text { Consumo abusivo } \\
\text { de álcool }\end{array}$}} & \multicolumn{4}{|c|}{ Geral } & \multicolumn{3}{|c|}{ Homens } & \multicolumn{5}{|c|}{ Mulheres } \\
\hline & & $\begin{array}{l}\text { RP Bruta } \\
\text { (IC 95\%) }\end{array}$ & $\mathrm{p}$ & $\begin{array}{l}\text { RP Ajustada }{ }^{a} \\
\quad \text { (IC 95\%) }\end{array}$ & $\mathrm{p}$ & $\begin{array}{l}\text { RP Bruta } \\
\text { (IC 95\%) }\end{array}$ & $\mathrm{p}$ & $\begin{array}{l}\text { RP Ajustada }{ }^{b} \\
\quad(\text { IC 95\%) }\end{array}$ & $\mathrm{p}$ & $\begin{array}{l}\text { RP Bruta } \\
\text { (IC 95\%) }\end{array}$ & $\mathrm{p}$ & $\begin{array}{l}\text { RP Ajustada }{ }^{b} \\
\text { (IC 95\%) }\end{array}$ & $\mathrm{p}$ \\
\hline \multirow[b]{2}{*}{ NAF } & Inativo & 1 & & 1 & & 1 & & 1 & & 1 & & 1 & \\
\hline & Ativo & $\begin{array}{c}1,3 \\
0,8-2,1\end{array}$ & 0,034 & $\begin{array}{c}1,1 \\
0,7-1,7\end{array}$ & 0,730 & $\begin{array}{c}2,3 \\
0,9-5,7\end{array}$ & 0,974 & $\begin{array}{c}2,2 \\
0,9-5,5\end{array}$ & 0,613 & $\begin{array}{c}0,9 \\
0,6-1,7\end{array}$ & 0,075 & $\begin{array}{c}0,8 \\
0,5-1,5\end{array}$ & 0,071 \\
\hline
\end{tabular}

$\mathrm{NAF}=$ Nível de atividade física; IC 95\%= Intervalo de confiança de 95\%; $\mathrm{p}=$ valor p regressão de Poisson; RP Ajustada; a= Razão de prevalência ajustada para sexo, idade e escolaridade; RP Ajustadab= Razão de prevalência ajustada para idade e escolaridade.

do conjunto da amostra, os ativos tiveram associação 1,5 vezes maior $(R P=1,5 ; \mathrm{IC} 95 \%: 1,2-1,9)(p=0,001)$ quando comparados aos inativos. $\mathrm{Na}$ comparação por sexo, os homens ativos apresentaram associação pelo menos 1,7 vezes maior $(R P=1,7$; IC95\%: $1,2-2,5)(p=$ $0,005)$ e as mulheres ativas apresentaram associação $1,4$ vezes maior (IC95\%: $1,1-1,8)(\mathrm{p}=0,033)$, quando comparados aos inativos. A associação permaneceu significativa após o ajuste para o sexo, idade e escolaridade. Para o consumo abusivo de álcool, os ativos apresentaram associação significativamente positiva de 1,3 vezes maior (IC95\% 1,3-2,1) ( $\mathrm{p}=0,034)$ quando comparados aos inativos, porém essa associação não permaneceu significante após o ajuste por sexo, idade e escolaridade.

\section{Discussão}

Este estudo forneceu para esta amostra, associação positiva do nível de AF de lazer com o consumo regular de bebida alcoólica. A prevalência do consumo de bebida alcoólica foi maior para homens quando comparados às mulheres. Apresentou tendência não linear de elevação para os maiores níveis de escolaridade e idade. Para o conjunto da amostra, foi detectado maior percentual de relatos de consumo regular de bebida alcoólica entre os fisicamente ativos, quando comparados aos inativos.

A diferença de gênero para o consumo de bebida alcoólica é relevante para a Saúde Pública ${ }^{18}$, vez que pode direcionar as ações de intervenção e de prevenção do uso nocivo do álcool com tratativas diferenciadas de gênero. Embora os homens tenham relatado maior consumo de bebida alcoólica, as mulheres tendem ser mais vulneráveis aos efeitos do álcool quando comparadas aos homens ${ }^{19}$.

A menor capacidade das mulheres para diluir o álcool devido a menor concentração de água corporal, a menor concentração de enzimas que processam o álcool antes de atingir a corrente sanguínea (desidrogenase), a maior taxa de estrogênio e o maior percentual de tecido adiposo corporal (gordura não absorve álcool), resulta na menor capacidade de metabolizar o álcool, fazendo com que as mulheres experimentem intoxicação por álcool mais rapidamente do que os homens ${ }^{20}$. Muito embora as mulheres enfrentem mais perigos do uso abusivo de álcool do que os homens, as mulheres são mais prováveis de pedir ajuda e de manter a abstinência quando tratadas adequadamente ${ }^{19}$.

De modo semelhante a este estudo, pesquisa conduzida pelo American College Health Association, envolveu 26.685 universitários e mostrou uma curva de dose-resposta positiva na associação do nível da AF com o consumo regular de bebida alcoólica ${ }^{21}$. Outra análise com mais de 40.000 adultos consumidores moderados de álcool de ambos os sexos (8-14 doses/semana para homens e 4-7 doses/semana para mulheres), resultou que os consumidores de álcool apresentaram pelo menos duas vezes mais probabilidade de serem fisicamente ativos quando comparados aos abstêmios ${ }^{22}$. No entanto, naqueles dois estudos apresentados, nenhuma conclusão de causalidade foi estabelecida, assim como não se estabeleceu a existência de prejuízo à saúde devido o consumo moderado de bebida alcoólica entre os mais ativos.

Os resultados deste estudo determina associação positiva do nível de AF com o consumo regular de bebida alcoólica, porém a associação não se confirmou para o consumo abusivo após ajustes por sexo, idade e escolaridade, contrariando os resultados do estudo do Behavioral Risk Factor Surveillance System (BRFSS) de 2005, após ter analisado 230.856 estadunidense e mostrado que bebedores abusivos (46 doses ou mais de bebidas no último mês para mulheres e 76 doses 
ou mais para homens) se exercitavam mais durante a semana quando comparados aos abstêmios e beber de modo leve, moderado e pesado se associou ao aumento de $9 \% ; 14,3 \%$ e $13,7 \%$, respectivamente, para a probabilidade de se exercitar vigorosamente ${ }^{23}$.

Curiosamente, pouco do que se sabe efetivamente sobre os mecanismos desta relação foi obtido em estudos com modelos animais, não sendo claro se os resultados se aplicam necessariamente aos seres humanos. Por questões éticas há poucos estudos de intervenção em humanos. A maior parte das investigações tem foco observacional e parecem revelar a maior força de associação entre os homens quando comparados às mulheres, principalmente após os 45 anos de idade ${ }^{24}$. A tendência do aumento de consumidores de bebida alcoólica em relação ao aumento da idade também foi observada nesse estudo.

A relação do aumento do consumo de bebida alcoólica com o aumento da idade, curiosamente ocorre justamente quando se observa uma tendência de decréscimo dos níveis de $\mathrm{AF}^{24,25}$ na população. Assim, pessoas com mais idade tendem a beber mais e a realizar menos AF. Estes consumidores de maior idade podem ser impulsionados a beberem mais por motivos alheios à AF, refletindo uma tendência motivacional extrínseca e responsável por ambas as atividades.

Efeitos específicos do álcool e da AF no cérebro têm sido utilizados para explicar, em parte, este fenômeno. A plausibilidade estaria relacionada ao mecanismo de recompensa que ocorre na área tegmental ventral e nucleusaccumbens do cérebro ${ }^{16}$. A modulação da recompensa e a experiência do prazer acontecem a partir de um suposto encadeamento de neurônios que interagem no sistema límbico por meio de diversos neurotransmissores - entre eles a serotonina, dopamina, norepinefrina e endorfina ${ }^{16}$. Os efeitos sobrepostos do álcool e da AF sobre os circuitos de recompensa neural podem competir parcialmente. Assim, dependendo da redução da concentração sanguínea desses neurotransmissores a sensação de bem-estar pode ser suplantada e motivar a procura por forma similar de recompensa.

Os diferentes comportamentos sociais e culturais tem sido a base para justificar a associação positiva do maior consumo de bebida alcoólica entre os mais ativos. Determinadas características da personalidade influenciam tanto a procura por AF como a procura por bebida alcoólica. Pessoas ativas apresentam mais relações sociais e, por isso, tendem a consumir mais bebidas alcoólicas $^{26}$. Também, para alguns indivíduos, principalmente os mais escolarizados, realizar AF de lazer com frequência pode ser um modo de compensar o consumo extra de energia proveniente da bebida alcoólica ou para contrabalançar alguns de seus efeitos deletérios.

$O$ grande desafio que se apresenta às futuras investigações é identificar os limites dessa associação e conhecer se há comprometimentos nocivos à saúde das pessoas devido a este suposto incentivo. Ademais, a ciência tem avançado na discussão sobre o consumo não abusivo de bebida alcoólica e tem apontado efeito de proteção à saúde com o uso moderado de alguns tipos de bebidas alcoólicas como o vinho ${ }^{27}$.

Decididamente, o que não se pode perder de vista são as robustas evidências do efeito nocivo do uso abusivo de bebida alcoólica. Segundo o Instituto Nacional de Ciências e Tecnologias para Políticas Públicas do Álcool e Outras Drogas (INPAD), entre os anos de 2006 a 2012, 32\% dos adultos que bebiam (21,8 milhões de pessoas) referiram não terem sido capazes de parar de beber após começar, 10\% (6,6 milhões de pessoas) referiu lesões diretas ou indiretas devido ao consumo, 8\% (7,4 milhões de pessoas) admitiram prejuízo que o uso de álcool causou ao trabalho, sendo que 4,9\% (4,6 milhões de pessoas) perderam ao menos um emprego devido ao consumo de bebida alcoólica e 9\% (12,4 milhões de pessoas) vivenciou efeito prejudicial na família ou no relacionamento íntimo. Somente no ano de 2012, este consumo esteve associado a 20\% dos casos de violência infantil, $50 \%$ dos casos de violência doméstica, $55 \%$ nos crimes de homicídio, $75 \%$ nas brigas com agressão física e $24 \%$ nas tentativas de suicídio. Além disso, 25\% dos bebedores não admitiram problema para dirigir quando os efeitos da bebida alcoólica estão apenas começando ${ }^{28}$.

Como limitações do presente estudo, é importante ponderar que medidas indiretas de $\mathrm{AF}$, como utilizada pelo sistema Vigitel podem não corresponder ao real nível de AF individual. Medidas diretas são consideradas mais precisas, todavia o custo é mais elevado e são impraticáveis em amostra numerosa como neste estudo. Os resultados desta análise se restringem ao domínio da AF de lazer. Assim, não se aplica a todos os outros domínios da AF. Os elementos característicos da AF de lazer como a ludicidade e a espontaneidade podem influenciar ou ser influenciados de modo diferente aos elementos característicos de outros domínios da AF, necessitando cautela na comparação direta desses resultados.

O consumo de bebida alcoólica é concebido pela opinião pública como um hábito potencialmente danoso, podendo o participante não ter respondido ao entrevistador com honestidade sobre o seu real consumo de álcool. 
Neste sentido, certo grau de viés de classificação pode estar presente, por exemplo, alguns bebedores abusivos podem ter sido categorizados como bebedores regulares. Portanto, a classificação de consumo regular e abusivo neste estudo foi utilizada para representar o menor e o maior comportamento nocivo do espectro de beber.

Em decorrência de limitações metodológicas do inquérito original do Vigitel, este estudo não foi capaz de explicar as diferenças de associação do nível de AF por tipo de bebida, teor alcoólico e composição química da bebida, embora se aceite que essas características estejam amplamente misturadas no consumo relatado pelos entrevistados.

Como em todos os estudos observacionais, não foi possível estabelecer temporalidade na relação entre consumo de bebida alcoólica e o nível de AF. Finalmente, o uso de entrevistas mediante telefone fixo não considera as pessoas que não possuem esse serviço. $\mathrm{Di}$ ferenças socioeconômicas entre as pessoas e localidades podem determinar variação na cobertura da rede de telefonia fixa. $\mathrm{O}$ uso de pesos pós-estratificados procurou minimizar possíveis diferenças entre a população total e a população do estudo.

Os resultados desse estudo sugerem associação positiva entre o consumo de bebida alcoólica e o nível de AF de lazer, no entanto, não sugerem que esta associação seja necessariamente prejudicial à saúde de todos os indivíduos. Mesmo diante da associação positiva, estes resultados devem ser utilizados com cautela no sentido de não promover a redução ou o abandono da prática regular de $\mathrm{AF}$ de lazer no âmbito populacional. A associação positiva, aqui encontrada, carece de uma melhor compreensão dos motivos que ligam esses comportamentos e as diferenças que ocorrem entre os sexos. As lacunas no conhecimento torna importante identificar com estudos adicionais, se essa associação está ou não agregada a comportamento temporalmente descontínuo, ou seja, se as pessoas envolvidas em AF bebem mais do que o habitual nos mesmos dias em que se envolvem com a AF ou em dias separados.

\section{Conflito de interesses}

Os autores declaram não haver conflito de interesses.

\section{Financiamento}

Universidade Estadual do Oeste do Paraná/Governo do Estado do Paraná.

\section{Contribuição dos autores}

Lima DF participou da concepção inicial do estudo, redação e análise crítica do texto. Luiz $\mathrm{OC}$, foi responsável pela construção do método do estudo, Silva MP, foi responsável pela análise estatística e análise dos resultados, Junior OM, Lima LA, Sampaio AA, participaram da revisão e análise crítica do texto.

\section{Referências}

1. Leasure JL, Neighbors C. Impulsivity moderates the association between physical activity and alcohol consumption. Alcohol. 2014;48(4):361-6.

2. Barry AE, Piazza-Gardner AK. Drunkorexia: understanding the co-occurrence of alcohol consumption and eating/ exercise weight management behaviors. J Am Coll Health. 2012;60(3):236-43.

3. Conroy DE, Ram N, Pincus AL, Coffman DL, Lorek AE, Rebar AL, Roche MJ. Daily Physical Activity and Alcohol Use Across the Adult Lifespan. Health Psychology. 2015;34(6):653-660.

4. Silva DAS. Nível de atividade física e fatores associados em acadêmicos de educação física de uma universidade pública do nordeste do Brasil. Rev Bras Ativ Fis Saúde. 2011;16(3):193-8.

5. Dodge T, Clarke P, Dwan R. The Relationship Between Physical Activity and Alcohol Use Among Adults in the United States: A Systematic Review of the Literature. Am J Health Promot. 2017;31(2):97-108.

6. Piazza-Gardner AK, Barry AE. Examining physical activity levels and alcohol consumption: are people who drink more active? Am J Health Promot. 2012;26(3):95-104.

7. Musselman JRB, Rutledge PC. The incongruous alcoholactivity association: Physical activity and alcohol consumption in college students. Psychol Sport Exerc. 2010;11(6):609-18.

8. Brasil. Ministério da Saúde. Instituto Nacional do Câncer. Inquérito domiciliar sobre comportamentos de risco e morbidade referida de doenças e agravos não transmissíveis: Brasil, 15 capitais e Distrito Federal, 2002-2003. Rio de Janeiro: INCA, 2004.

9. Naimi TS, Stockwell T, Zhao J, Xuan Z, Dangardt F Saitz $\mathrm{R}$. Selection biases in observational studies affect associations between 'moderate' alcohol consumption and mortality. Addiction. Debate. 2017;112(2):207-14.

10. Haskell WL, Lee IM, Pate RR, Powell KE, Blair SN, Franklin BA, et al. Physical Activity and Public Health: Updated Recommendation for Adults from the American College of Sport Medicine and the American Heart Association. Med Sci Sport Exerc. 2007;3(2):1423-34.

11. Pan American Health Organization, Word Health Organization. Regional Status Report on Alcohol and Health in the Americas. Washington, DC: PAHO, 2015.

12. World Health Organization. Global status report on alcohol and health 2014. Geneva: WHO, 2014.

13. Hallgren M, Vancampfort D, Giesen ES, Lundin A, Stubbs B. Exercise as treatment for alcohol use disorders: systematic review and meta-analysis. $\mathrm{Br} \mathrm{J}$ Sports Med. 2017;51(14):1058-64.

14. Leasure JL, Nixon K. Exercise neuroprotection in a rat model of binge alcohol consumption. Alcohol Clin Exp Res. 2010;34:404-14.

15. Leasure JL, Neighbors C. Impulsivity moderates the association between physical activity and alcohol consumption. Alcohol. 2014;48(4):361-6.

16. Sander D, Grandjean D, Pourtois G, Schwartz S, Seghier $\mathrm{ML}, \mathrm{Scherer} \mathrm{KR}$ et al. Emotion and attention interactions in social cognition: brain regions involved in processing anger prosody. Neurolmage. 2005;28(4):848-58. 
17. Brasil. Ministério da Saúde. VIGITEL - Brasil 2014: Vigilância de Fatores de Risco E Proteção para Doenças Crônicas por Inquérito Telefônico. Brasília (DF): Ministério da Saúde; 2015.

18. Loch MR, Souza RKT. Capital Social: dados descritivos de estudo de base populacional e associação com comportamentos simultâneos de risco à saúde. Rev Bras Ativ Fis Saúde. 2014;19(6):774-76.

19. Wolle CC, Sanches M, Zilberman ML, Caetano RZ, Laranjeira M, Ilana P. Differences in drinking patterns between men and women in Brazil. Rev Bras Psiquiatr. 2013;33(4):367-73.

20. Centro de Informações sobre Saúde e Álcool. Compreensão da concorrência de comportamentos como consumo de álcool, transtornos alimentares, prática de atividades físicas a partir da drunkorexia. CISA, São Paulo, 2013. [Citado 2013 jul 08]. Disponível em: http://www.cisa.org.br/index.php.

21. Smothers B, Bertolucci D. Alcohol consumption and healthpromoting behavior in a US household sample: leisure-time physical activity. J Stud Alcohol. 2001;62(4):467-76.

22. Sinyor D, Brown T, Rostant L, Seraganian P. The role of a physical fitness program in the treatment of alcoholism.J Stud Alcohol. 1992;43(3):380-6.

23. French MT, Popovici I, Maclean JC. Do alcohol consumers exercise more? Findings from a national survey. Am J Health Promot. 2009;24(1):2-10.
24. Friederichs SA, Bolman C, Oenema A, Lechner L. Profiling physical activity motivation based on self-determination theory: a cluster analysis approach. BMC Psychol. 2015;3(1):1-8.

25. Lima DF. Atividade física de adultos nas capitais brasileiras e no Distrito Federal: um estudo transversal. Tese. (Doutorado em Medicina Preventiva). Faculdade de Medicina. Universidade de São Paulo. São Paulo, 2014.

26. Cooke R, Dahdah M, Norman P, French DP. How well does the theory of planned behaviour predict alcohol consumption? A systematic review and meta-analysis. Health Psychol Rev. 2016;10(2):148-67.

27. Chiva-Blanch G, Arranz S, Lamuela-Raventos RM, Estruch R. Effects of Wine, Alcohol and Polyphenols on Cardiovascular Disease Risk Factors: Evidences from Human Studies. Alcohol and Alcoholism. 2013;48(3):270-7.

28. Laranjeira R. II Levantamento Nacional de Álcool e Drogas (LENAD) - 2012. Ronaldo Laranjeira (Org.), São Paulo: Instituto Nacional de Ciência e Tecnologia para Políticas Públicas de Álcool e Outras Drogas (INPAD), UNIFESP, 2014.

\section{Como citar este artigo:}

Lima DF, Silva MP, Júnior OM, Lima LA, Sampaio AA, Luiz OC. Associação da atividade física de lazer com o consumo de bebidas alcoólicas em adultos. Rev Bras Ati Fis Saúde. 2017;22(6):576-83. DOI: 10.12820/rbafs.v.22n6p576-83. 